\title{
Design and Parameters Optimization of the Fractional Order Anti-windup Controller for Multileaf Collimator
}

\author{
Xin Zhang ${ }^{1, *}$, Jiaxin $\mathrm{Li}^{1}$, Jianwu Dang ${ }^{1}$, Zhiqiang Liu ${ }^{2}$ and Yongzhi Min ${ }^{3}$ \\ ${ }^{1}$ School of Automation \& Electrical Engineering, Lanzhou Jiaotong University, Lanzhou 730070, China \\ ${ }^{2}$ Gansu Provincial Cancer Hospital, Lanzhou 730050, China \\ ${ }^{3} 317$ A Carnegie Library, Mathematic Department, Syracuse University, Syracuse, NY, 13244, United States
}

Received 9 January 2017; Accepted 15 March 2017

\begin{abstract}
During the conformal radiotherapy, the multileaf collimator is usually used to block radiation to avoid the radiation in the normal tissue around the tumor for precise irradiation in target areas. However, there are several shortcomings in the ability of rapidity and anti-interference of precise leaf control in terms of the traditional controllers of leaf position for multileaf collimator. To ensure the rapidity of leaf position in place for short treatment time, a design method of controller of leaf position for multileaf collimator has been proposed, which can keep the leaves in place with short time as well as high accuracy. First, the motor that drives the leaf movement of multileaf collimator was taken as the controlled object, and the corresponding motor model was established. Second, the design method of the fractional order anti-windup controller of leaf position was proposed according to the controlled object. Finally, using quantum-behaved particle swarm optimization (QPSO) to ensure the accuracy of leaf position for multileaf collimator in place, the parameters of the controller of leaf position for multileaf collimator were optimized. The simulation results show that the rising and settling time of the output response of the leaf drive unit is $1.31 \mathrm{~s}$ in the controller of leaf position for multileaf collimator using the proposed design method, which is better than the $6.4 \mathrm{~s}$ in the proportional integral differential (PID) algorithm based on the particle swarm optimization (PSO) and the $12.55 \mathrm{~s}$ in the fuzzy PID algorithm respectively. While the parameter tuning of the controller of leaf position for multileaf collimator is performed by the QPSO algorithm, the required iteration number is less. In addition, after adding the interference, the proposed controller can make the system recover to the much more stable operation state with a stronger ability of anti-interference. The proposed method provides great reference value for the research on the precise leaf position of multileaf collimator and the cooperation control strategy in the later stage.
\end{abstract}

Keywords: Particle swarm optimization, Fractional order anti-windup, Parameter optimization, Radiation therapy, Multileaf collimator

\section{Introduction}

With the further development of radiotherapy technology in China, the research on the improvement of radiotherapy equipment has also been greatly developed, and the accurate target positioning and the dose correction are of critical importance [1]. The main purpose of developing the multileaf collimator is to achieve conformal radiotherapy, which accurately illuminates the lesion target [2]. The radiotherapy system addresses the following main problems: medical image segmentation, medical volume data structure based on body tissue contour, three-dimensional surface reconstruction algorithm based on medical volume data, three-dimensional dose calculation model, and the system control of multileaf collimator [3]. The multileaf collimator is controlled to enable the leaf to reach the predetermined position accurately, improve the dose accuracy at each point within the control irradiation field, and reduce the complications of surrounding normal tissue, and effectively

*E-mail address: zhangx@mail.lzitu.cn

ISSN: 1791-2377 @ 2017 Eastern Macedonia and Thrace Institute of Technology. All rights reserved. improve the gain ratio of treatment in the radiation therapy. Therefore, during the radiotherapy process, the movement of each leaf for multileaf collimator and the position accuracy of the leaves are precisely controlled. To control the multileaf collimator regardless of the method adopted, three elements must be considered: the leaf drive unit, the real-time monitoring of leaf position, and the control logic of leaf movement. The leaf drive unit of electric multileaf collimator includes two types of motors, namely digital or analog ones. The measure of monitoring leaf position involves the use of mechanical limit switches, optical imaging systems, and linear encoders. The control logic of leaf involves the determination of leaf speed and the leaf position in consideration of the dose compensation. For multileaf collimator, the choice of leaf drive unit [4] and the design of the controller of drive unit are the first problems affecting the precise leaves in place. In addition, the design of drive unit controller needs to consider the rapidity of leaf in place to shorten treatment time [5],[6] and the ability of antiinterference to avoid friction and collision between the leaves [7], which affect the accuracy of leaf position in place. The parameters tuning of the controller of leaf position determines the accuracy of leaf position in place, which 
directly affects the treatment effect. It should be urgently addressed to implement the precise control of leaf position for multileaf collimator. Therefore, the research on the control accuracy of leaf position has some practical significance.

\section{State of the art}

The leaf of multileaf collimator is a mechanical part that has strict requirements for acceleration, speed, and position. If the acceleration, speed, and position of the leaf were controlled precisely, that can achieve the goal of the treatment plan. In the case of the shortest treatment time, the selection of the leaf drive unit and the design of the drive unit of the leaf position controller are essential to achieve the maximum gain ratio of the radiation therapy treatment. Domestic and foreign scholars have conducted substantial research on the multileaf collimator leaf drive unit selection problem. Yao used a miniature stepper motor for the leaf drive unit [8], but in practical applications, if the acceleration and speed of the drive unit are high, the stepper motor is easily out of step-by-step control itself, thereby destroying the conformal treatment effect. Yun used permanent magnet servo motor to drive the motion of the multileaf collimator leaf [9]. DC servomotors have very good speed performance and fast dynamic response, that can be quickly and accurately controlled with speed-position sensors. However, with the passage of time, micropermanent magnet DC servomotors show a fatal weakness as DC motors due to the high current (relative to the micromotor) and high-speed frequent variable speed operation that cause the motor mechanical commutation and brush to produce serious sparks and serious contact surface wear, which result in frequent malfunctions that require continuous repair and replacement. Thus, the use of a new type of drive actuator instead of a miniature permanent magnet DC servomotor and stepper motor is imperative. The brushless DC motor with electronic devices instead of the mechanical brush with speed-position sensors can achieve fast and precise control [10]. Zaferullah designed a double closed-loop controller of the drive unit for the leaf of multileaf collimator based on the field-programmable gate array (FPGA) [11], but Zaferullah did not mention which controller was used for each closedloop system. Jia-li designed the proportional integral differential (PID) closed-loop controller to realize the precise control of the leaf movement displacement and speed for multileaf collimator based on C8051F023 and NJM3771D2 [12], but the author did not address the tuning of the parameters of the PID controller. PID control has a long history in the industrial control development process [13], and now PID controller is still widely used in industry control realm. The parameter selection and tuning of PID controller play crucial roles in the control effect. Xin used the weighted particle swarm optimization (PSO) algorithm to tune the parameters of the PID controller to ensure that the precision of the multileaf collimator leaf satisfies the radiotherapy requirements [10]. However, the fitness value of the PSO generated by the algorithm does not satisfy the requirement as shown in the experimental results. The method easily becomes premature and does not obtain the global optimal solution. The windup phenomenon still occurs, due to the existence of the integral part [14]. Therefore, a brushless DC motor is used as the driving unit of the multileaf collimator in this study, and the fractional order anti-windup controller is designed based on the fractional order control theory [15],[16], considering the phenomenon of integral saturation in the actual control process. The controller ensures the rapidity of the leaf in place and its anti-interference capability. To address the difficulty in controller parameter optimization, quantumbehaved particle swarm optimization (QPSO), which is highly versatile method, is used to optimize the parameters of the designed controller.

The remainder of this study is organized as follows. Section 3 described the working principle of the multileaf collimator and establishes the model of the drive unit. The leaf position of the fractional order anti-windup controller was designed for multileaf collimator. Section 4, in the same range of parameters, presented the verification of the designed controller using experiments and the comparison with other methods. The conclusions were summarized in Section 5.

\section{Methodology}

\subsection{Control system model of multileaf collimator}

The multileaf collimator is mainly composed of leaves, which are arranged in pairs. In the radiation therapy system, the positions of the multileaf collimator leaves are determined based on the shape of the tumor. The shape of the rays that pass through the multileaf collimator is similar to the projection on the irradiated surface of the tumor. The diagram of the working principle of multileaf collimator is shown in Fig. 1 and Fig. 2.

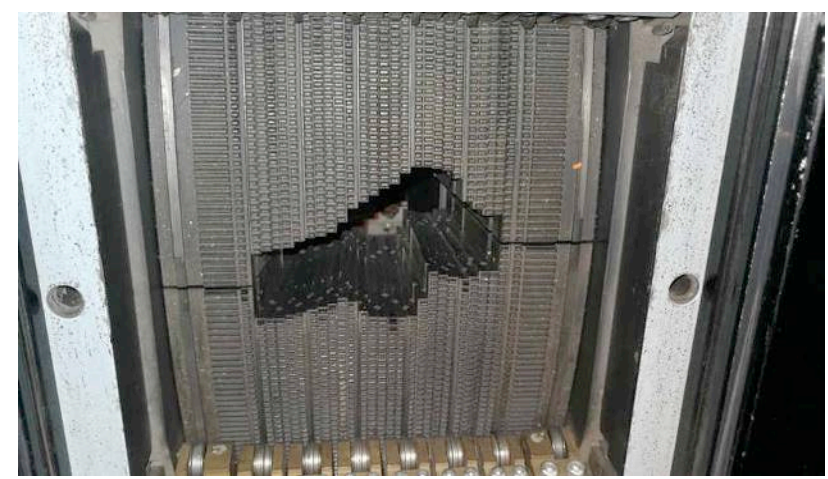

Fig. 1. Principle of conformal radiotherapy for multileaf collimator

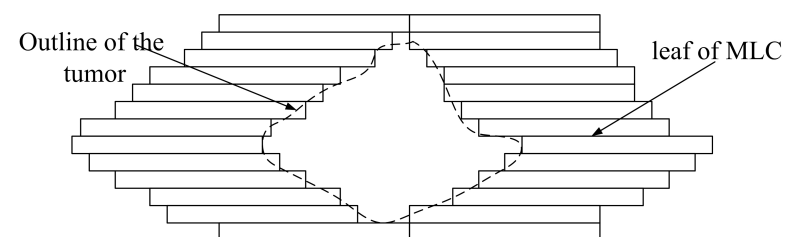

Fig. 2. Working principle diagram of multileaf collimator

In practical applications, each leaf is driven by a microelectric motor, and the rotational movement of the motor is converted to a linear motion of the leaf through a screw rod. Each leaf has a set of independent controller. The electric motor used in this system includes stepping motor, DC servomotor, and brushless DC motor. The brushless DC motor (BLDC motor) is widely used in the multileaf collimator control system and has many unique advantages. The electrical connection diagram of the multileaf collimator drive unit is shown in Fig. 3. The structure diagram of the close-loop leaves position control system is shown in Fig. 4. 
To ensure the accuracy of the leaves in place, the following methods are used: (1) accurate speed control of the drive motor, acceleration, and number of turns or number of steps to achieve the accuracy of leaves position control (this requires the use of encoders to detect the operating status of the motor); and (2) direct detection of the position of the leaves [17] and identification the next running requirements of the motor (commonly used methods in twodimensional ionization chamber matrix to verify the multileaf collimator leaves position precision). Substantial study on motor control has been conducted. The BLDC motor can be controlled precisely through the drive file of multileaf collimator that is generated by the radiation treatment planning system and contains the location information for each leaf.

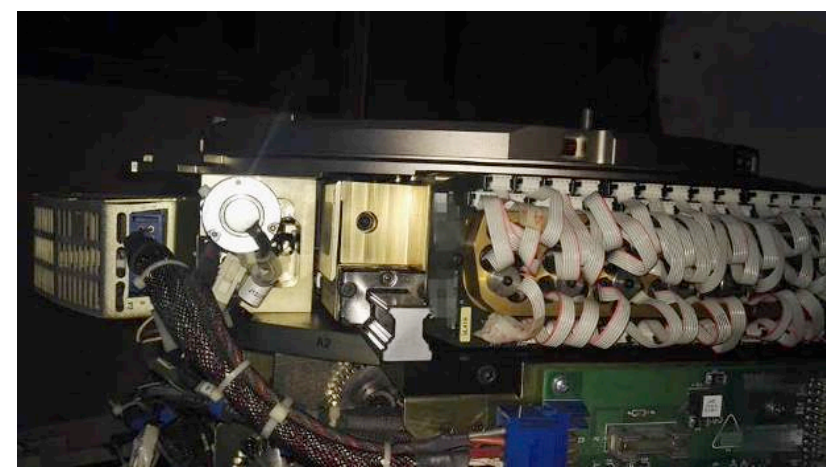

Fig. 3. Electrical connection diagram of multileaf collimator drive unit

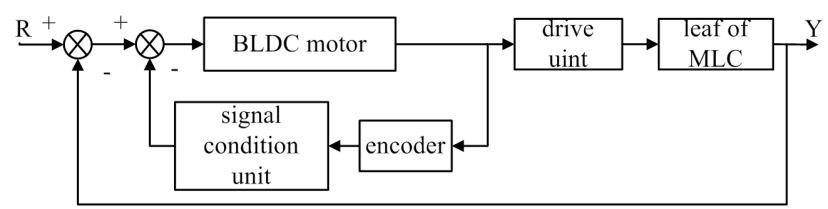

Fig. 4. Structure diagram of the close-loop position control system

The characteristic equations of the BLDC motor can be represented as follows [18]:

$$
\begin{aligned}
& v_{\text {app }}(t)=L \frac{d i(t)}{d t}+R i(t)+v_{\text {emf }}(t) \\
& v_{\text {emf }}=K_{\mathrm{b}} \omega(t) \\
& T(t)=K_{\mathrm{t}} i(t) \\
& T(t)=J \frac{d \omega(t)}{d t}+D \omega(t)
\end{aligned}
$$

where $v_{\text {app }}$ is the applied voltage, $\omega(t)$ is the motor speed, $L$ is the inductance of the stator, $i(t)$ is the current of the circuit, $R$ is the resistance of the stator, $v_{\text {emf }}$ is the back electromotive force, $T$ is the torque of the motor, $D$ is the viscous coefficient, $J$ is the moment of inertia, $K_{\mathrm{t}}$ is the motor torque constant and $K_{\mathrm{b}}$ is the back electromotive force constant. From the characteristic equations of the BLDC motor, the transfer function of the speed model is obtained using Eq. (5):

$$
G(s)=\frac{Y(s)}{R(s)}=\frac{K_{t}}{L J^{2}+(L D+R J) s+K_{\mathrm{t}} K_{\mathrm{b}}}
$$

The parameters of the motor used for simulation are as follows:

$R$ is the resistance of the stator: $1.26 \Omega$;

$K_{\mathrm{t}}$ is the motor torque constant: $3.08 \times 10^{-3} \mathrm{~N} \cdot \mathrm{m} / \mathrm{A}$;

$L$ is the inductance of the stator: $0.035 \mathrm{mH}$;

$J$ is the moment of inertia: $0.75 \mathrm{~g} \mathrm{~cm}^{2}$.

From the above parameters, the back electromotive force constants are $K_{b}=9.55, K_{t}=0.0294$ and the viscous coefficient is $D=\frac{K_{t} K_{b}}{R_{a}}=7.14 \times 10^{-5}$.

The parameters are substituted into Eq. (5): $G(s)=\frac{118.46}{s^{2}+36346.15 s+3.5}$.

\subsection{Review of Quantum-Behaved Particle Swarm Optimization}

\subsubsection{Particle swarm optimization}

PSO is an evolutionary computation technique that was presented by Kennedy and Eberhart in 1995 [19]. The idea originated from the social behavior of biology in nature, such as flocks of birds and schools of fish.

In the PSO algorithm [20],[21], each particle in a swarm represents a solution to the problem and is defined by its position and velocity. The mathematical description of the basic PSO is as follows.

Supposing the scale of the swarm is $N$, the position of particle $i$ can be expressed as Eq. (6).

$x_{i}=\left(x_{i 1}, x_{i 2}, \cdots, x_{i D}\right)$

The velocity of the particle is defined as the distance of the particle movement in each iteration and described as Eq. (7).

$v_{i}=\left(v_{i 1}, v_{i 2}, \cdots, v_{i D}\right)$

The velocity of particle $i(i=1,2, \cdots, N)$ in $d(d=1,2, \cdots, D)$-dimensional space, which is adjusted according to Eq. (8), is

$$
\begin{gathered}
v_{i d}=v_{i d}+c_{1} \operatorname{rand}_{1}()\left(p_{i d}-x_{i d}\right)+c_{2} \operatorname{rand}_{2}()\left(p_{g d}-x_{i d}\right) \\
\left\{\begin{array}{ccc}
v_{i d}=v_{\max }, & \text { if } & v_{i d}>v_{\max } \\
v_{i d}=-v_{\max }, & \text { if } & v_{i d}<-v_{\max }
\end{array}\right.
\end{gathered}
$$

Finally, the particle can adjust its position according to Eq. (9):

$x_{i d}=x_{i d}+v_{i d}$

where $N$ is the number of particles in the group, $d$ is the dimension, $v_{i d}$ is the velocity of particle , $c_{1}$ and $c_{2}$ are the acceleration constants, $\operatorname{rand}()$ is a random number between 0 and $1, x_{i d}$ is the current position of particle $i$, 
$p_{i d}$ is the best previous position of the $i$ th particle, and $p_{g d}$ is the best particle among all the particles in the population.

\subsubsection{Quantum-Behaved Particle Swarm Optimization}

Quantum-behaved particle swarm optimization [22], which is based on the delta potential well, considers that particles have quantum behavior. In the process of optimization, the particles move in the delta potential well that is centered on the best position $p$. In the delta potential well, the quantum potential can be described as $V(x)=-\lambda \delta(x-p)$, $y=x-p$. The quantum state equation can be expressed as $\psi(y)=\frac{1}{\sqrt{L}} e^{-|y| / L}$. The probability density function of the particle in position $y$ is $Q(y)=|\psi(y)|^{2}=\frac{1}{L} e^{-2|y| / L}$.

To calculate the fitness value of particles, Monte Carlo simulation is used to calculate the precise position of particles. Supposing $s \in(0,1 / L)$ is a random number, $s=(1 / L) \operatorname{rand}(0,1)=\frac{1}{L} u, u=\operatorname{rand}(0,1)$.

The position equation of the particle can be obtained as follows:

$x=p \pm \frac{L}{2} \ln (1 / u)$

$L$ is defined as $L(t+1)=2 \beta \mid$ mbest $-X(t) \mid$, and mbest is defined as the average optimal position of all particles:

$$
\text { mbest }=\frac{1}{M} \sum_{i=1}^{M} P_{i}=\left(\frac{1}{M} \sum_{i=1}^{M} P_{i 1}, \frac{1}{M} \sum_{i=1}^{M} P_{i 2}, \cdots, \frac{1}{M} \sum_{i=1}^{M} P_{i D}\right)
$$

In the formula above, $M$ is the number of particles, $D$ is the dimension of the particle, and $P_{i}$ is the $P_{b e s t}$ of the ith particles: $P_{i d}=\left(\varphi_{1} P_{i d}+\varphi_{2} P_{g d}\right) /\left(\varphi_{1}+\varphi_{2}\right)$, where $\varphi_{1}$ and $\varphi_{2}$ are uniformly distributed random numbers on $[0,1]$ and $P_{g}$ is the optimal value of the population.

Finally, the evolution equation of the QPSO algorithm is obtained as follows:

$$
\begin{aligned}
& X(t+1)= \\
& \left\{\begin{array}{l}
P_{i d}-\beta \cdot \mid \text { mbest }_{d}-X_{i d}(t) \mid \cdot \ln (1 / u), u>0.5 \\
P_{i d}+\beta \cdot \mid \text { mbest }_{d}-X_{i d}(t) \mid \cdot \ln (1 / u), u \leq 0.5
\end{array}\right.
\end{aligned}
$$

$\beta$ is the contraction expansion coefficient:

$\beta=0.5 \cdot \frac{\text { MAXITER }-t}{\text { MAXITER }}+0.5$

where MAXITER is the maximum number of iterations and $t$ is the current iterations number of particle.

\subsection{Design of fractional order anti-windup controller based on QPSO}

3.3.1 Design of fractional order anti-windup controller

This study combines the anti-windup [23],[24] and fractional order PID to form the position controller of the multileaf collimator leaves. The state transition condition of the controller is realized by the judgment of the integral state $q$.
It is represented as Eq. (14), and the structure diagram of the fractional order anti-windup control system is shown in Fig. 5.

$$
q^{\prime}=\left\{\begin{array}{cc}
K_{i} e(t) & u_{n}=u_{s} \\
-\frac{1}{\tau} q & u_{n}>u_{s}
\end{array}\right.
$$

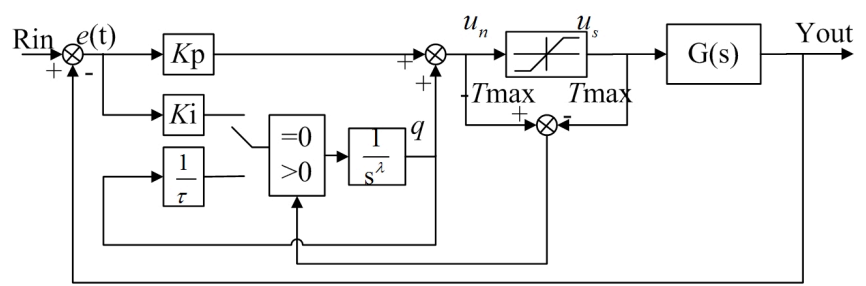

Fig. 5. Structure diagram of the fractional order anti-windup control system

\subsubsection{Design of fractional order anti-windup controller based on QPSO}

The structure diagram of the fractional order anti-windup control system based on QPSO is shown in Fig. 6.

This controller is designed mainly for the following two components: the fractional order anti-windup controller for the object and the module of the QPSO algorithm.

According to the operating state of the system, the QPSO module can optimize the parameters of the fractional order anti-windup controller to satisfy the performance requirements, and the output of this module will provide the optimized parameter of the fractional order anti-windup controller.

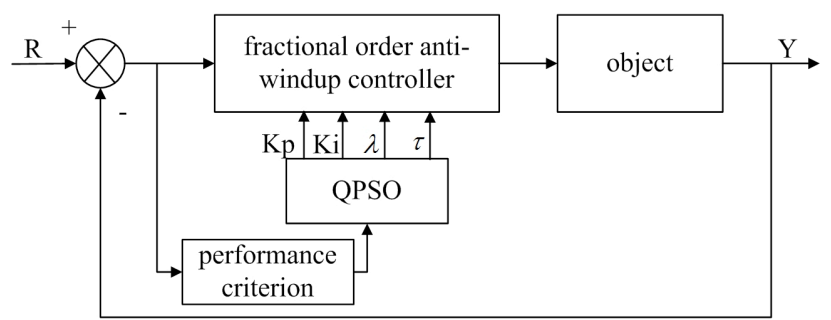

Fig. 6. Structure diagram of fractional order anti-windup control system based on QPSO

The most common performance criteria in fractional order anti-windup controller are described as follows:

(1) integration of time weight square error:

ITSE $: J=\int_{0}^{\infty} t^{2} e^{2}(t) d t$

(2) the integral of time multiplied by the absolute error:

IATE $: J=\int_{0}^{\infty}|e(t) t d t|$

(3) integration of squared error:

ISE $: J=\int_{0}^{\infty} e^{2}(t) d t$

(4) integration of absolute error:

$I A E: J=\int_{0}^{\infty}|e(t)| d t$ 
IATE: $J=\int_{0}^{\infty}|e(t) t d t|$ was selected as the performance criteria of the fractional order anti-windup controller based on QPSO in this study.

\subsubsection{Implementation of fractional order anti-windup controller based on QPSO}

The implementation steps of the fractional order anti-windup controller parameter optimization based on QPSO are as follows:

Step 1: Generate initial population;

Step 2: Determine the fitness value of each particle using the performance criteria;

Step 3: Analyze the fitness value of each particle and update the global optimum position value;

Step 4: Update the velocity and position of the particle;

Step 5: If the maximum iteration number is reached or the performance criteria is satisfactory, the system obtains the optimal solution. Otherwise, the steps are repeated from step 2.

The specific method is as follows:

(1) Initialize the number of the particle (population size). Population size affects the performance of the QPSO algorithm. The local optimal solution is easy to obtain if the population size is small. If the population size is too large, it is difficult and time-consuming to obtain and will increase the complexity of the algorithm exponentially. In this study, the population size is set to 50 .

(2) Initialize the particle dimension. The particle dimension is determined by the optimized object. The output of the QPSO module are the three parameters of the fractional order anti-windup controller, namely, $K_{\mathrm{p}}, K_{\mathrm{i}}$, and the $\lambda$, and the $\tau$ of the particle dimension is set to 4 .

(3) Initialize the range of particles. To accelerate the calculation speed, the PID parameters have been adjusted by Ziegler-Nichols with trial and error firstly. Then, $K_{\mathrm{p}}$ and $K_{\mathrm{i}}$ are set to $K_{\mathrm{p}} \in(0,1500), K_{\mathrm{i}} \in(0,3), \lambda \in(0,2)$ and $\tau \in(0,2)$.

(4) Determine the fitness value. Calculate the fitness value of each particle through the integral of time multiplied by the absolute error, IATE: $J=\int_{0}^{\infty}|e(t)| t d t$, and take the minimum fitness value of 0.1 .

Finally, the initial values of the particles are randomly generated within a certain space. The other parameters are chosen as follows: $\omega$ (inertia weight factor) $=0.6 ; c_{1}$ and $c_{2}=2 ; v_{\max }=1, v_{\min }=-1 ;$ iteration $=10$.

\subsubsection{Fractional order anti-windup controller} experimental platform

The leaf position controller experiment platform is shown in Fig. 7. The experimental system uses TI's motor control special digital signal processor TMS320F2812 as the control core. The power-driven module consists of a single-phase diode bridge rectifier, a large capacitance filter, and threephase two-level IGBT inverter components, and the incremental photoelectric encoder is used to detect motor speed.

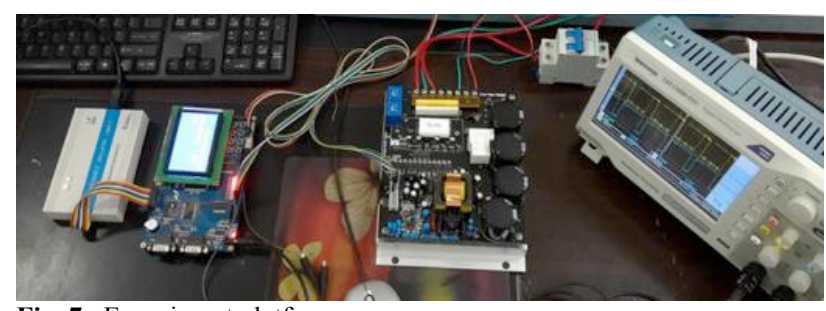

Fig. 7. Experiment platform

\section{Result Analysis and Discussion}

The design takes the step signal as the input signal to achieve the step response of the control system. Examples of fuzzy adaptive PID and PSO-PID controllers are provided to illustrate the effectiveness of the proposed design. The output response obtained by the leaf driving unit is shown in Fig. 8. The best fitness values of the QPSO and PSO are shown in Fig. 9.

Compared with the results obtained using the PSO and the fuzzy control method to optimize the parameters of the PID controller, the method adopted in this study has no overshoot when the two parameters have the same selection ranges $\left(K_{\mathrm{p}} \in(0,1500)\right.$ and $\left.K_{\mathrm{i}} \in(0,3)\right)$, as shown in Fig. 8 . However, the results obtained by the proposed method are superior to the results of the PSO-PID and the fuzzy-PID control methods based on the comparison of the two performance indices of the rising and stabilizing times. In the fifth iteration, the proposed method obtains the fitness value that satisfies the requirement according to the performance index evaluation function in Eq. (16) as shown in Fig. 9. The optimal solution for the controller parameter is determined, and the number of iterations is small. The performance indexes of the system response results obtained by the three methods are shown in Table 1 .

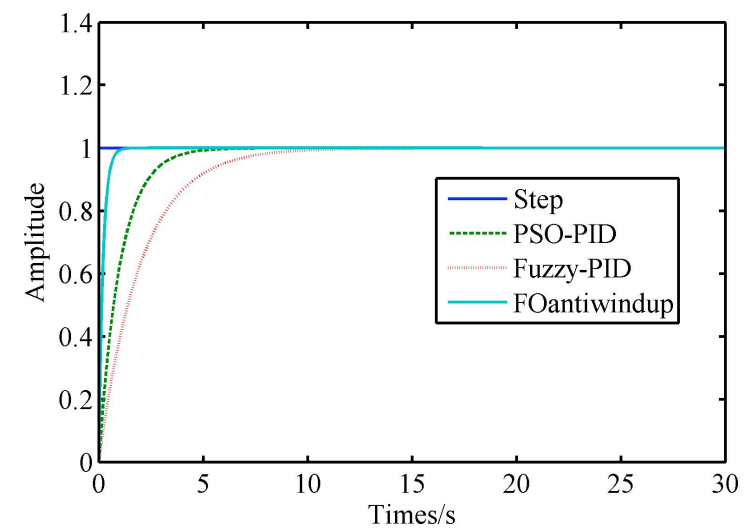

Fig. 8. Comparison of output response results obtained by the leafdriven unit

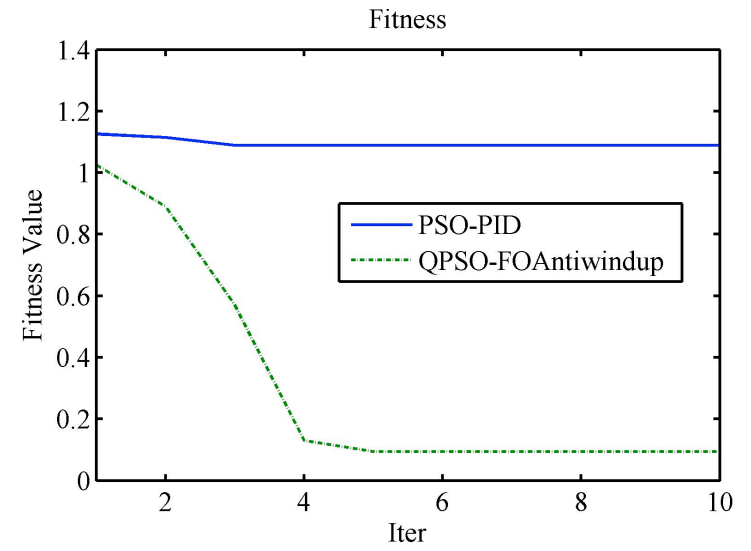

Fig. 9. Comparison curves of fitness values of two PSO 
Table 1. Comparison of Performance Results of the System Response Results in Three Methods

\begin{tabular}{c|c|c|c}
\hline Index & Pethod & PSO-PID & Fuzzy-PID \\
\hline Overshot (\%) & $0 \%$ & $0 \%$ & $0 \%$ \\
\hline Rise time (s) & $1.31 \mathrm{~s}$ & $6.4 \mathrm{~s}$ & 12.55 \\
\hline Settling time (s) & $1.31 \mathrm{~s}$ & $6.4 \mathrm{~s}$ & 12.55 \\
\hline
\end{tabular}

Table 1 shows that the proposed method allows the leaf to reach a predetermined position quickly, approximately $1.31 \mathrm{~s}$, thereby shortening the treatment time. To verify strong anti-interference ability of the proposed method, that can avoid the leaf position error caused by leaves collision. Given the step signal and the leaf drive unit stable operation (20s), the leaf drive unit increases the load simulation of the impact of leaves collision. The results of the three methods are shown in Fig. 10.

After adding interference, the output has minimum fluctuations and no oscillation exists. The time required to return to the steady state is the shortest, and the antiinterference ability is stronger than the other two methods as shown in Fig. 10.

Using the experimental platform shown in Fig. 7, the other parameters in the experiment are set as follows. The PWM sampling period is $T_{s}=200 \mu \mathrm{s}$, the speed control period is $10 T_{s}$, and the speed set point is $n=500 \mathrm{rad} / \mathrm{min}$. The experimental data can be transmitted to the host PC through the serial communication port of the TMS320F812. The measured results of the motor output speed are shown in Fig. 11. The measured results of the motor speed have the same physical meaning as the simulation results obtained in Fig. 8. The experimental results verify the effectiveness of the proposed leaf position controller design and parameter optimization method.

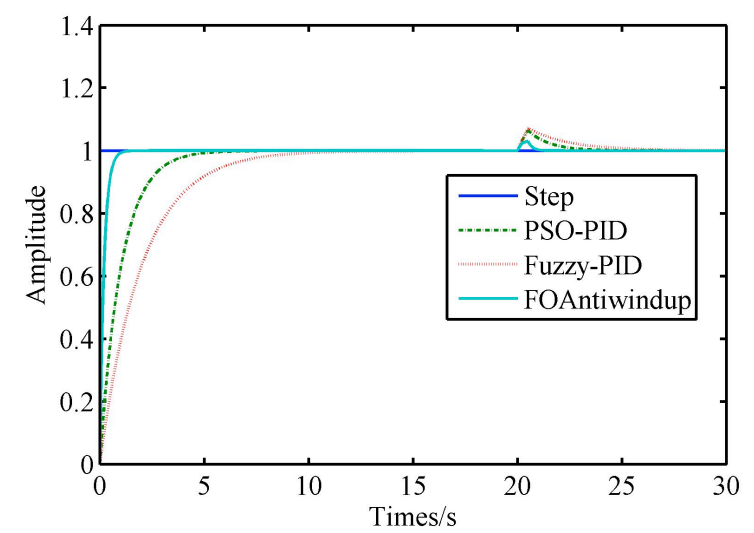

Fig. 10. Step-response curve of output with the three control strategies with interference

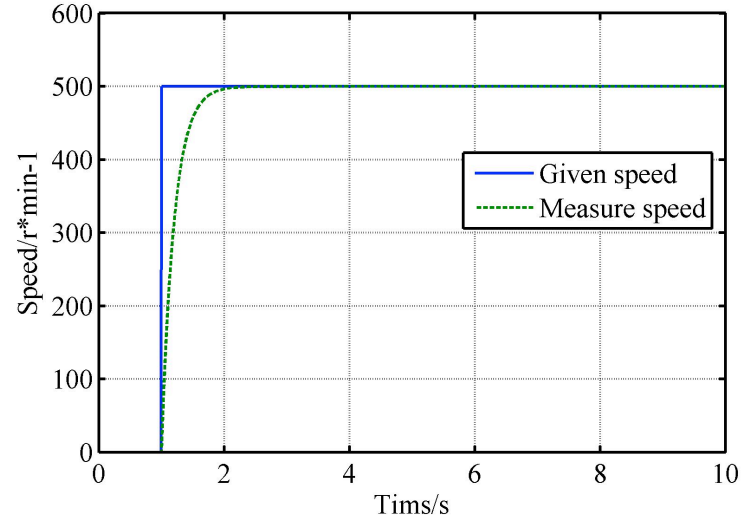

Fig. 11. Measured dynamic response curve

\section{Conclusions}

To solve the problem of multileaf collimator, in which the leaf does not reach the predetermined position quickly, the research on the design method of the controller of leaf position for multileaf collimator was done. The brushless DC motor that drives the leaf movement was taken as the controlled object. Combined with fractional order control theory and anti-windup controller, a fractional order antiwindup controller of leaf position for multileaf collimator was designed. The parameters of the proposed controller were optimized by the intelligent optimization algorithm of QPSO The final conclusions are as follows:

(1) The global optimal solution of controller parameters of leaf position can be obtained quickly using QPSO, which improves the ability of global search for particle swarms.

(2) The proposed fractional order anti-windup controller based on QPSO of leaf position for multileaf collimator enables the output response of the leaf drive unit to quickly reach the given value and completely satisfy the requirement of shortening the treatment time.

(3) The proposed method can quickly eliminate the leaf position error due to collision caused by leaves and enable the leaf drive unit to have the strong ability of antiinterference.

Based on the control theory and intelligent optimization algorithm, the fractional order anti-windup position controller has been designed for a single leaf of multileaf collimator. In addition, the proposed method is universal, which lays the foundation for the further research on the multileaf cooperation control for multileaf collimator. However, the digital implementation of the proposed method is relatively complex. The proposed method will be transplanted into FPGA in the future work.

\section{Acknowledgements}

The study was supported by the National Natural Science Foundation of China (Grant No. 61562057), the Program for Changjiang Scholars and Innovative Research Team in University (IRT_16R36) and the Lanzhou Jiaotong University Youth Foundation (No.2013041).

This is an Open Access article distributed under the terms of the Creative Commons Attribution Licence

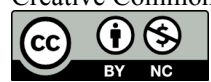




\section{References}

1. Thorwarth, Daniela, Geets, X., Paiusco, M., "Physical radiotherapy treatment planning based on functional PET/CT data". Radiotherapy and Oncology, 96(3), 2010, pp.317-324.

2. Guptaa, T.,Agarwalb, J., Jaina, S., Phurailatpama, R., Kannana, S., Ghosh-Laskarb, S., Murthya, V., Budrukkarb, A., Dinshawb, K., Prabhashb, K., Chaturvedib, P., Cruzb, A. "Three-dimensional conformal radiotherapy (3D-CRT) versus intensity modulated radiation therapy (IMRT) in squamous cell carcinoma of the head and neck: a randomized controlled trial". Radiotherapy and Oncology, 104(3), 2012, pp.343-348.

3. Arumugam, S., Xing, A., Goozee, G., \& Holloway, L. "Independent calculation-based verification of IMRT plans using a 3D dosecalculation engine". Medical dosimetry, 38(4), 2014, pp.376-384.

4. Lamey, M., Yun, J., Burke, B., Rathee, S., \& Fallone, B. G. "Radio frequency noise from an MLC: a feasibility study of the use of an MLC for linac-MR systems". Physics in medicine and biology, 55(4), 2010, pp.981.

5. Popescu, C.C, Olivotto, I.A., Beckham, W.A., Ansbacher, W., Zavgorodni, S., Shaffer, R., Wai, E.S.,Otto, K. "Volumetric modulated arc therapy improves dosimetry and reduces treatment time compared to conventional intensity-modulated radiotherapy for locoregional radiotherapy of left-sided breast cancer and internal mammary nodes". International Journal of Radiation Oncology Biology Physics, 76(1), 2010, pp.287-295.

6. Thames, H.D., Kuban, D., Levy, L.B., Horwitz, E.M., Kupelian, P., Martinez, A., Michalski, J., Pisansky, T., Sandler, H., Shipley, W., Zelefsky, M., Zietman, A. "The role of overall treatment time in the outcome of radiotherapy of prostate cancer: an analysis of biochemical failure in 4839 men treated between 1987 and 1995". Radiotherapy and Oncology, 96(1), 2010, pp.6-12.

7. Oliver, M., Gagne, I., Bush, K., Zavgorodni, S., Ansbacher, W., \& Beckham, W. "Clinical significance of multi-leaf collimator positional errors for volumetric modulated arc therapy". Radiotherapy and oncology, 97(3), 2010, pp.554-560.

8. Yao, C. H., Chang, T. H., Tsai, M. J., Lai, Y. C., Chen, Y. A., Chang, Y. J., \& Chen, C. H. "Dose verification of volumetric modulation arc therapy by using a NIPAM gel dosimeter combined with a parallel-beam optical computed tomography scanner". Journal of Radioanalytical and Nuclear Chemistry, 311(2), 2017, pp.1-10.

9. Yun, J., St Aubin, J., Rathee, S., Fallone, B.G. "Brushed permanent magnet DC MLC motor operation in an external magnetic field". Medical physics, (37)5, 2010, pp.2131-2134.

10. Xin, Z. "Parameter Optimization of PID Controller Based on PSO for Multi-leaf Collimator". Indonesian Journal of Electrical Engineering and Computer Science, 11(10), 2013, pp.6127-6134.

11. Zaferullah, Z., Mishra, B. K., Bansode, R., Vidwans, M., \& Dsouza, K. "Control of Collimator for Conformal Radiation Therapy based on FPGA Implementation". Global Journal of Research In Engineering, 14(4), 2014, pp.27-31.
12. Jia-li, Y. A. N., Dong, W. A. N. G., Rui-fen, C. A. O., Gang, S. O. N. G., \& Yi-can, W. U. "The Design of Leaf Control Module of Multi-leaf Collimator Based on C8051F023". Science Technology and Engineering, 13(12), 2013, pp.3401-3405.

13. Tan, W. "Unified tuning of PID load frequency controller for power systems via IMC". IEEE Transactions on power systems, 25(1), 2010, pp.341-350.

14. Kothare, M. V., Campo, P. J., Morari, M., \& Nett, C. N. "A unified framework for the study of anti-windup designs". Automatica, 30(12), 1994, pp.1869-1883.

15. Podlubny, I., "Fractional-Order Systems and $P I^{\lambda} D^{\mu}$-Controllers". IEEE Transactions on Automatic Control, 44(1), 1999, pp.208-214.

16. Podlubny, I., Petras, I., Vinagre, B.M., Chen, Y.Q., O’Leary, P., Dorcak, L. "Realization of fractional order controllers". Acta Montanistica Slovaca (S1335-1788), 8(4), 2003, pp.233-235.

17. Page, R.F., Abbott, N.L., Davies, J., Dyke, E.L., Randles, H.J., Velthuis, J.J., Fletcher, S., Gregory, S.D., Hall, C., John, A., Lawrence, H., Stevens, P.H., Hugtenburg, R.P., Tunbridge, V. "Using a Monolithic Active Pixel Sensor for Monitoring Multileaf Collimator Positions in Intensity Modulated Radiotherapy". IEEE Transactions on Nuclear Science, 61(1), 2014, pp.71-74.

18. Fang, J., Li, H., \& Han, B. "Torque ripple reduction in BLDC torque motor with nonideal back EMF". IEEE transactions on power electronics, 27(11), 2012, pp.4630-4637.

19. Eberhart, R. C., and J. Kennedy. "Particle swarm optimization", proceeding of IEEE International Conference on Neural Network, Perth, Australia: IEEE, 1995, pp.1942-1948.

20. Ishaque, Kashif, and Zainal Salam. "A deterministic particle swarm optimization maximum power point tracker for photovoltaic system under partial shading condition". IEEE transactions on industrial electronics, 60(8), 2013, pp.3195-3206.

21. Renaudineau, H., Donatantonio, F., Fontchastagner, J., Petrone, G., Spagnuolo, G., Martin, J. P., \& Pierfederici, S. "A PSO-based global MPPT technique for distributed PV power generation". IEEE Transactions on Industrial Electronics, 62(2), 2015, pp.1047-1058.

22. Pradhan, Smita, and Dipti Patra. "RMI based non-rigid image registration using BF-QPSO optimization and P-spline". AEUInternational Journal of Electronics and Communications, (69)3, 2015, pp.609-621.

23. Shin, H. B., \& Park, J. G. “Anti-windup PID controller with integral state predictor for variable-speed motor drives". IEEE Transactions on Industrial Electronics, 59(3), 2012, pp.1509-1516.

24. Herrmann, G., Menon, P. P., Turner, M. C., Bates, D. G., \& Postlethwaite, I. "Anti-windup synthesis for nonlinear dynamic inversion control schemes". International Journal of Robust and Nonlinear Control, 20(13), 2010, pp.1465-1482. 